\title{
A novel ceramic coating for reduced metal ion release in metal-on-metal hip surgery
}

\author{
Gordon W. Blunn, ${ }^{1 \dagger}$ Roberta Ferro De Godoy, ${ }^{1}$ Jayantilal Meswania, ${ }^{1}$ Tim W. R. Briggs, ${ }^{2}$ Philippa Tyler, ${ }^{3}$ \\ Rikin Hargunani, ${ }^{3}$ Hannah Wilson, ${ }^{4}$ Imran Khan, ${ }^{4}$ Tim Marriott, ${ }^{4}$ Melanie J. Coathup ${ }^{1 *}$ \\ ${ }^{1}$ Institute of Orthopaedics and Musculoskeletal Science, Division of Surgery and Interventional Science, University College \\ London, The Royal National Orthopaedic Hospital, Stanmore, Middlesex, HA7 4LP, UK \\ ${ }^{2}$ Royal National Orthopaedic Hospital, Stanmore, Middlesex, HA7 4LP, UK \\ ${ }^{3}$ Department of Radiology, Royal National Orthopaedic Hospital Trust, Stanmore, Middlesex, HA7 4LP, UK \\ ${ }^{4}$ Zimmer Biomet, Dorcan Industrial Estate, Swindon, Wiltshire, UK
}

Received 1 June 2018; revised 12 September 2018; accepted 30 September 2018

Published online 00 Month 2018 in Wiley Online Library (wileyonlinelibrary.com). DOI: 10.1002/jbm.b.34268

\begin{abstract}
An ovine total hip arthroplasty model was developed to evaluate metal ion release, wear, the biological response and adverse tissue reaction to metal-on-metal (MoM) bearing materials. The performance of an advanced superlattice ceramic coating (SLC) was evaluated as a bearing surface and experimental groups divided into; (1) MoM articulating surfaces coated with a SLC coating (SLC-MoM), (2) uncoated MoM surfaces (MoM), and (3) metal on polyethylene (MoP) surfaces. Implants remained in vivo for 13 months and blood chromium ( $\mathrm{Cr}$ ) and cobalt (Co) metal ion levels were measured pre and postoperatively. Synovial tissue was graded using an ALVAL scoring system. When compared with the MoM group, sheep with SLC-MoM implants showed significantly lower levels of chromium and cobalt metal ions within blood over
\end{abstract}

the 13-month period. Evidence of gray tissue staining was observed in the synovium of implants in the MOM group. A significantly lower ALVAL score was measured in the SLCMoM group (3.88) when compared with MoM components (6.67) $(p=0.010)$. ALVAL results showed no significant difference when SLC-MOM components were compared to MoP (5.25). This model was able to distinguish wear and the effect of released debris between different bearing combinations and demonstrated the effect of a SLC coating when applied onto the bearing surface. (C) 2018 Wiley Periodicals, Inc. J Biomed Mater Res B Part B: 00B: 000-000, 2018.

Key Words: total hip replacement, wear, MoM, ARMD, ceramic coating, cobalt, chromium, ovine model

How to cite this article: Blunn GW, De Godoy RF, Meswania J, Briggs TWR, Tyler P, Hargunani R, Wilson H, Khan I, Marriott T, Coathup MJ. 2018. A novel ceramic coating for reduced metal ion release in metal-on-metal hip surgery. J Biomed Mater Res $B$ Part B. 2018:00:00:1-12.

\section{INTRODUCTION}

Total hip arthroplasty (THA) is considered one of the most successful procedures in modern surgery and metal-onmetal (MoM) THA was commonly used over the last two decades mainly in young and active patients with arthritis. However MoM THA, hip resurfacing as well as modular head-neck and neck-stem components with a range of bearing materials have resulted in an increase of local and circulating metal products resulting in high short term failure rates. $^{1-3}$ Adverse soft tissue reactions in patients with a MoM hip replacement are associated with cobalt (Co) and chromium (Cr) particles released from the implant. ${ }^{3-5}$ MoM bearings generate approximately $6.7 \times 10^{12}-2.5 \times 10^{14}$ nano-scale (mean size of $30 \mathrm{~nm}$ ) particles per year. This is 13,500-times the number of ultra-high molecular weight polyethylene particles produced from a typical MoP bearing. ${ }^{6}$ The smaller particles result in a lower volumetric wear than MoP hips but wider particle dissemination, beyond the peri-prosthetic tissues. ${ }^{6}$ Micromotion at the interface between modular junctions and between the female taper (head component) and male taper (stem component), facilitates mechanically assisted crevice (MACC) and fretting corrosion, leading to the production of further amounts of wear debris and metal ion release. ${ }^{7,8}$ Corrosion around the headneck interface is considered a significant source of metal release. $^{9-11}$ An adverse tissue reaction can result in the development of pseudotumors, which can be granulomatous or cystic lesions, neither infective nor neoplastic and develop in the vicinity of the THA. They can be large or small in size with or without communication to the joint often causing

†Present address: School of Pharmacy and Biomedical Sciences, University of Portsmouth, St Michael's Building, White Swan Road, Portsmouth, PO1 2DT, UK

Present address: College of Medicine, University of Central Florida, Health Sciences Campus, 6900 Lake Nona Blvd, Orlando, FL, USA. Tel: +1 (407) 266 7184. e-mail: melanie.coathup@ucf.edu.

Correspondence to: M. J. Coathup; e-mail: melanie.coathup@ucf.edu 
pain, swelling, pressure effects, subluxation, bone, and softtissue destruction and can as well be asymptomatic. ${ }^{12}$ A cell mediated type IV hypersensitivity reaction has also been described, aseptic lymphocytic vasculitis-associated lesions (ALVAL) is a term coined to describe an intense perivascular lymphatic infiltration that occurs around certain MoM devices. ${ }^{13}$ Although ALVAL is not unique to tissues surrounding MoM implants, the intensity of lymphocytic infiltrate is greater in association with metal debris from MoM implants than with other bearing surfaces. ${ }^{13,14}$ MoM hip implants are now rarely used however advantages in the use of larger bearings remain, including improved range of motion, ${ }^{15}$ reduced risk of revision and in the case of resurfacing devices, conservative bone-preserving surgery where less resection of femoral host bone is required. ${ }^{16,17}$.

The development of enhanced bearing surfaces engineered with superior wear characteristics may reduce wear debris formation and metal ion release in MoM hips. One potential methodology for achieving this is to combine the surface properties of a ceramic with the bulk properties of a metal. The following factors can contribute to improved bearing performance: (i) increased hardness of the components; (ii) surface chemistry that reduces adhesive friction; (iii) surface-coated components that remain undamaged and thus provide a smoother surface, leading to reduced wear, and (iv) increased corrosion resistance by encasing the metallic substrate in an inert ceramic layer. It is also possible that this surface treatment may reduce the release of $\mathrm{Cr}$ and Co ions by limiting wear, tribocorrosion and corrosion of the wear particles. Importantly, hip simulators failed to predict the biological response identified with certain designs of MoM bearings. For this reason, we have developed an ovine THA model where the metal ion release and biological response can be measured. A chromium nitride/niobium nitride $(\mathrm{CrN} / \mathrm{NbN})$ coating utilizing a nanoscale multilayer/superlattice (SLC) structure was applied onto $\mathrm{CoCr}$ bearing surfaces to increase hardness, wear and corrosion resistance. A high power impulse magnetron sputtering technology deposited dense and adherent coatings onto the bearing surfaces ${ }^{18}$ and the aim of the study was to use an animal model to investigate the performance of coated MoM bearings compared with non-coated MoM and MoP bearing combinations. Our hypothesis was that a SLC applied to the femoral head and acetabular cup surface would result in a reduction in volumetric wear, a decrease in blood chromium and cobalt ion levels and reduce any potential adverse reactions to metal debris (ARMD).

\section{MATERIALS AND METHODS}

Unilateral total hip replacement surgery was performed on the right hip of 15 skeletally mature Charolais rams aged 2-5 years and weighing between 90 and $120 \mathrm{~kg}$. All procedures were carried out following ethics approval granted by the Royal Veterinary College and in compliance with the United Kingdom Home Office regulations (Animal Scientific Procedures Act [1986]). Animals were randomly allocated into one of three experimental groups: (1) MoM articulating surfaces coated with a superlattice ceramic coating (SLCMoM), (2) uncoated MoM surfaces (MoM), and (3) metal on polyethylene (MoP) bearings. Three sets of implants (comprising a femoral head, acetabular cup and femoral stem) in each group were investigated and each remained in vivo for 13 months.

\section{Implant manufacture}

In all groups, cast cobalt chrome ( $\mathrm{CoCr}$ ) femoral heads and monobloc $\mathrm{CoCr}$ acetabular cups were manufactured by Mat Ortho Ltd (Leatherhead, Kent, United Kingdom). The femoral heads and acetabular shells used in the SLC-MoM group were coated by Zimmer-Biomet Ltd (Swindon, United Kingdom), where a $5 \mu \mathrm{m}$ multilayered $\mathrm{CrN} / \mathrm{NbN}$ superlatticestructured ceramic coating was applied using High Power Impulse Magnetron Sputtering technology (HIPIMS) (HTC 1000-4, four cathode system (Hauzer Techno Coatings, Europe B.V. Venlo, The Netherlands) that provided a highly dense, hard and adherent coating on the bearing surface. ${ }^{18}$ The superlattice structure consisted of repeating $\mathrm{CrN}$ and $\mathrm{NbN}$ layers and the advantage of applying a nano-layered, inhomogenous columnar shaped crystal structure is that the hardness values of the coating is improved combined with excellent corrosion behavior due to the presence of the highly electrochemically stable niobium as a partner material. CoCr implant stems were manufactured by CDRM Ltd. (High Wycombe, Bucks, United Kingdom) using a Direct Metal Laser Sintering technique followed by Hot Isostatic Pressing (HIPping) and heat treatment (Bodycote, Somerset, United Kingdom) (Figure 1). The length of the stems from shoulder to tip were $65 \mathrm{~mm}$ and tapered from $12 \mathrm{~mm}$ in width proximally down to $8 \mathrm{~mm}$ at the distal stem. The neck

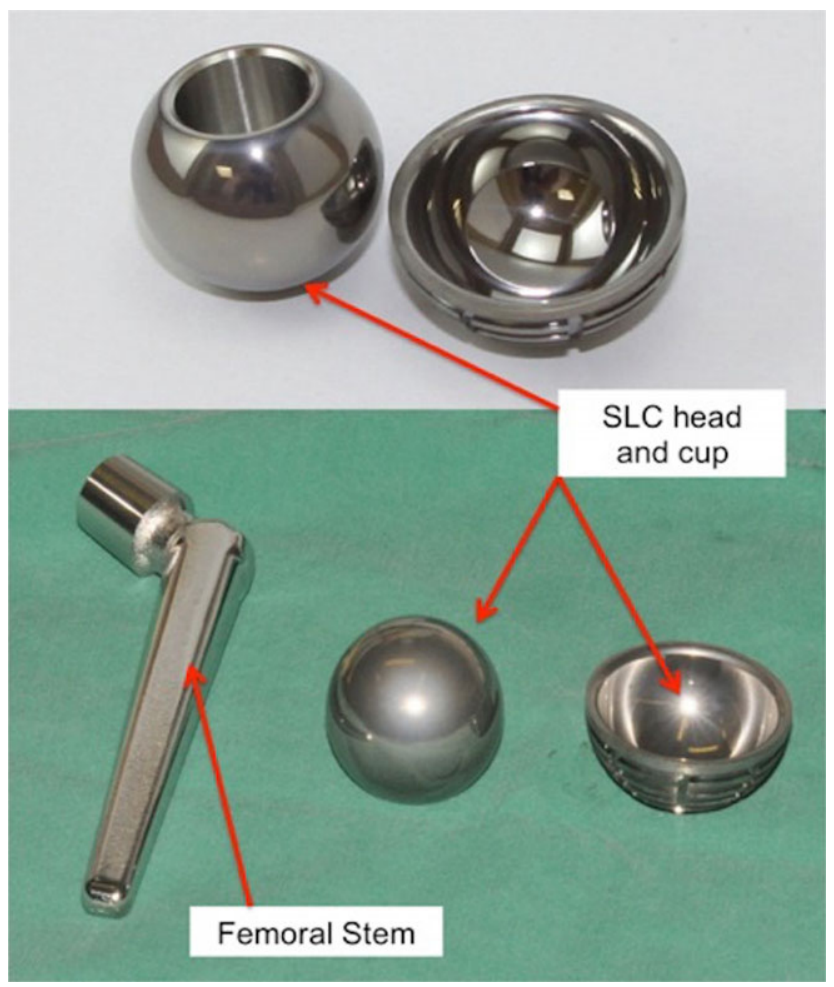

FIGURE 1. The femoral component with femoral head and acetabular cup prior to surgery and following application of the SLC. 


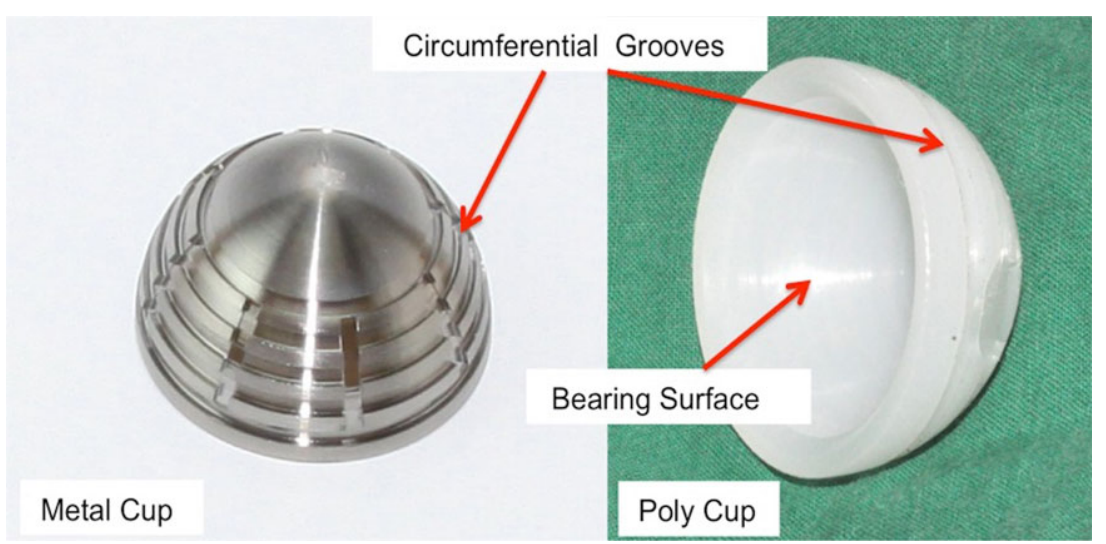

FIGURE 2. Photograph showing the position of anti-rotational grooves located on the back of acetabular cups.

angle was off-set at $45^{\circ}$ and stem surfaces were highly polished. All femoral stems were cemented in place. Implants were modular and mini $12 / 14$ tapers, $17 \mathrm{~mm}$ in length were manufactured to a surface finish of $R_{z} 6-20 \mu \mathrm{m}$. Both the male tapers located on the stem and female tapers within the femoral heads remained uncoated in all groups. All polyethylene cemented acetabular cups were manufactured using Medical Grade GUR 1050 ultra-high molecular weight polyethylene (Orthoplastics, Bacup, Lancashire, United Kingdom). Prior to implantation, components were gamma sterilized at an irradiation dose of 25 kGy (Synergy Health, Abergavenny, United Kingdom). The materials, design and manufacture of all implants conformed with relevant ISO Standards. Cobalt chrome femoral heads $(26 \pm 0.03 \mathrm{~mm}$ diameter) engaged onto the tapered femoral neck. All acetabular cups were manufactured to an inner diameter of $26.12 \pm 0.03 \mathrm{~mm}$ and an outer diameter of $30 \pm 0.03 \mathrm{~mm}$. The maximum radial clearance (pre-coating) was $+0.03-0.09 \mathrm{~mm}$. Cups were manufactured for cemented fixation and each designed with four equally positioned $1.5 \mathrm{~mm}$ wide and $0.7 \mathrm{~mm}$ deep circumferential grooves present to prevent rotation and enhance fixation of the cup within the cement mantle (Figure 2). The pre-operative surface roughness $\left(R_{a}\right)$ of the uncoated bearing surfaces was $0.015 \pm 0.001 \mu \mathrm{m}$ and $0.131 \pm 0.055 \mu \mathrm{m}$ in the SLC group.

\section{Surgical technique}

Under anesthesia an anterior lateral approach was used to access the hip joint and following dislocation a femoral neck osteotomy was performed. The femoral head was removed and the acetabulum reamed using a custom reamer of appropriate dimensions, until bleeding subchondral bone was observed. Using a $3 \mathrm{~mm}$ diameter drill, $4 \mathrm{~mm}$ deep holes were made in order to augment mechanical interlock of the cement mantle within the acetabulum. The acetabulum was then washed and dried prior to cementation of the acetabular cup using antibiotic loaded Refobacin ${ }^{\circledR}$ (Biomet Europe, Swindon, United Kingdom) cement. The femur was prepared by removing trabecular bone, inserting a cement restrictor and introducing bone cement into the femoral canal using a cement gun. The cement was pressurized, the femoral stem inserted, the hip reduced and the wound closed. Antibiotic and analgesic prophylaxis was administered daily with subcutaneous injections of Exenel ${ }^{\mathrm{TM}}$ (ceftiofur hydrochloride, $1 \mathrm{~mL} / 50 \mathrm{~kg}$; Pfizer, Tadworth, United Kingdom) and intramuscular injections of Vetergesic ${ }^{\mathrm{TM}}$ (buprenorphine, $0.6 \mathrm{mg} /$ animal; Reckitt and Coleman Products, Hull, United Kingdom) for 3 days post-operatively. Once fully recovered, animals were individually exercised by walking them in a track area within set boundaries for an hour a day, five times a week to maximize wear debris generation.

\section{Scanning electron microscopy}

Scanning electron microscopy (secondary electron and backscatter detection) was used to examine and compare component surfaces both prior to surgery and following retrieval at 13 months post-implantation (20 KeV electron beam (JSM35C; Jeol, Welwyn Garden City, United Kingdom)). Following retrieval and washing, metal femoral heads and cups were each sputter-coated with a layer of gold palladium prior to insertion into the SEM chamber.

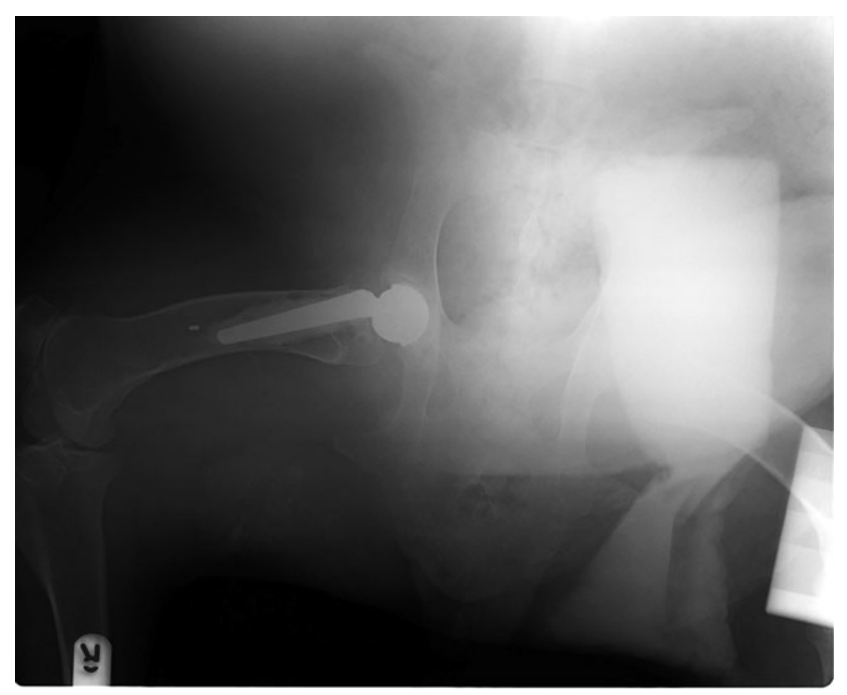

FIGURE 3. A radiograph showing a SLC-MoM total hip replacement of the right joint in a sheep lying supine and 6 months post-surgery. 


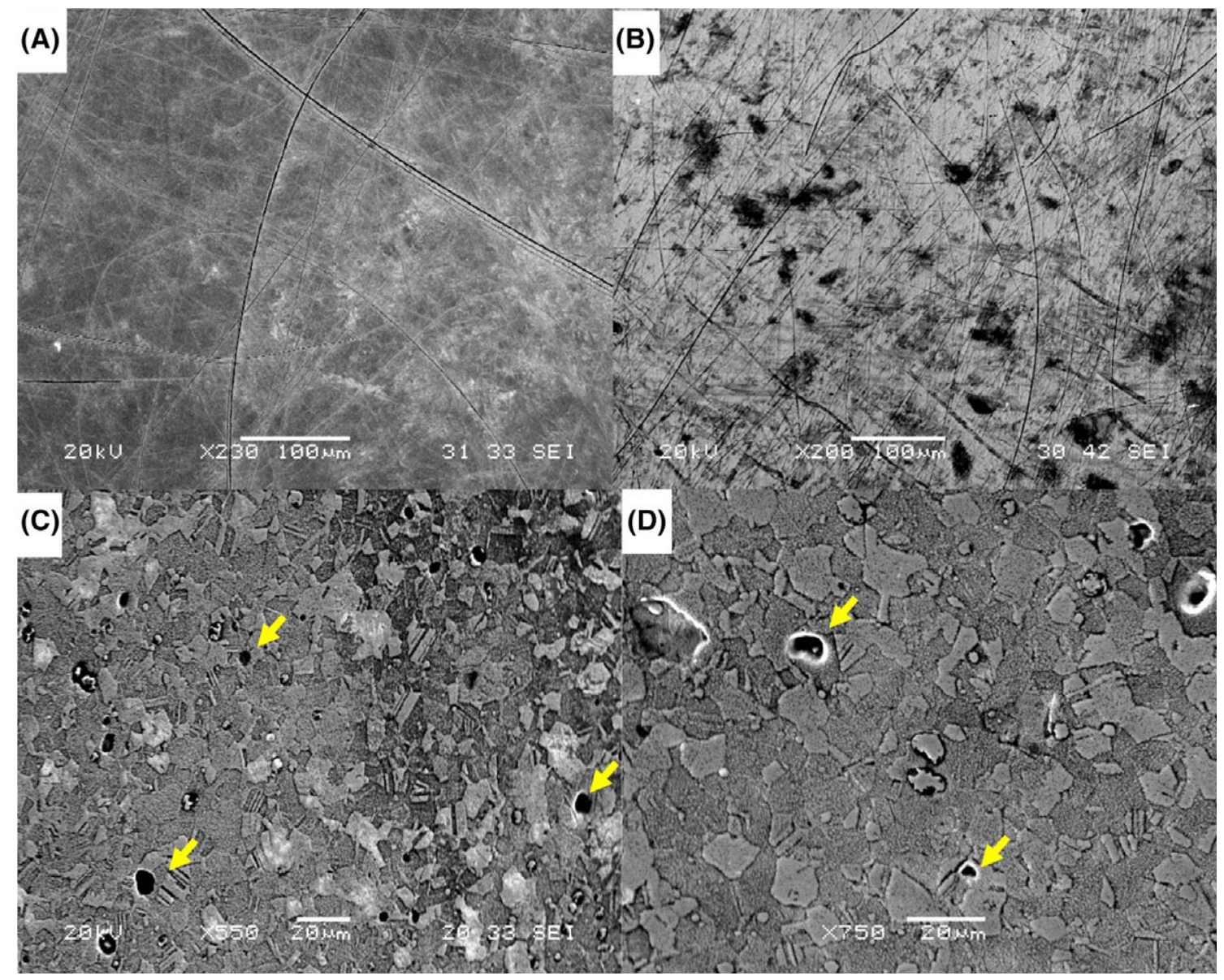

FIGURE 4. Electron micrographs showing (A) scratches on the surface of the femoral head in the MoP group (13 months post-surgery), (B) the MoM group (13 months post-op), with fewer scratches seen on (C) the SLC-MoM surface which was similar to (D) the pre-operative SLC-MoM surface. The arrows in 4 (C) and (D) indicate pin hole defects within the SLC coating formed during the coating application process. The dark patches seen in 4 (B), may be tribochemical reactions that have occurred on the implant surface.

\section{Co-ordinate measuring machine and Taylor Hobson} Talyrond high-precision roundness measuring machine Volumetric wear measurements of the bearing surfaces was assessed using a three-axis co-ordinate measuring machine
(CMM) before, following SLC application and after retrieval enabling volumetric wear to be assessed. Circumferential probe measurements were taken radially every $0.5 \mathrm{~mm}$ over the entire bearing surface of each femoral head and acetabular

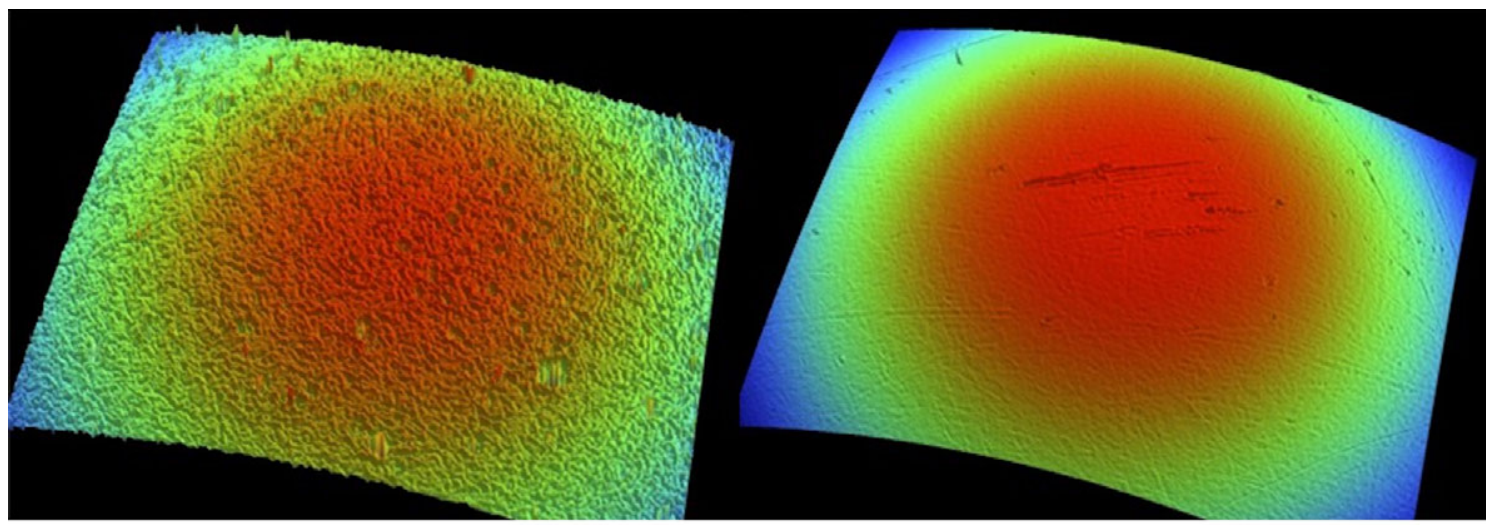

(A) SLC-MoM T0: Mean $\mathrm{R}_{\mathrm{a}} 0.131 \mu \mathrm{m} \pm$

(B) MoP T0: Mean $\mathrm{R}_{\mathrm{a}} 0.015 \mu \mathrm{m} \pm 0.001$ 0.055

FIGURE 5. A photo showing the contours measured using the CMM, from a femoral head in the MoM group before and following retrieval. 
cup. This resulted in 18,000-20,000 measurements taken for each component. A Taylor Hobson Talyrond High-Precision Roundness measuring machine was used to quantify all male and female taper surfaces prior to implantation and after retrieval. For the taper measurements, four equi-distant traces were taken along the length of the taper on the neck region of the femoral component and within the inner taper of the femoral head.

\section{Three-dimensional surface measurements}

Three-dimensional non-contact analyses of all femoral head and acetabular implants were undertaken using Optical Microscopy (Contour-GT, Bruker, MA). Four equi-distant measurements along the length of each of the taper surfaces on the femoral stem and within the femoral head were obtained. Five measurements were taken on each head and cup bearing surface, where one measurement was taken at the apex of the head (or cup) and four at equi-distant measurements around the periphery taken $30^{\circ}$ distal to the apex. Profile roughness measurements $\left(R_{a}\right.$, and $\left.R_{z}\right)$ were obtained and the means compared.

\section{Chromium and cobalt metal ion analysis}

Whole-blood samples were intravenously collected in traceelement blood tubes (K2EDTA; Becton, Dickinson and Co, Franklin Lakes, NJ, USA) using a Vacutainer 1 system (Becton, Dickinson and Co) pre-operatively and at 1, 3, 6, 9, 12, and 13 months post-operatively. These trace-element tubes were certified for analysis of metal ion blood concentrations. Samples were sent to the Supraregional Trace Element Laboratory, Imperial College Healthcare NHS Trust, United Kingdom for $\mathrm{Cr}$ and $\mathrm{Co}$ metal ion analysis at each of the time-points investigated. A mean of six readings/sample (ppb [ $\mu \mathrm{g} / \mathrm{L}])$ of whole blood were provided.

\section{Implant imaging}

Lateral radiographs taken post-operatively and at 1, 3, 6, 9, 12 , and 13 months post-surgery were assessed.

\section{Magnetic resonance imaging analysis}

Following euthanasia, bilateral pelvic MRI scans were performed using a Philips Achieva 1.5 Tesla Scanner. Sheep were placed supine on the scanner table and a SENSETM Surface body coil positioned over the hips. A metal artifact reduction sequence (MARS) protocol was used to reduce the size and intensity of artifact resulting from magnetic field distortion caused by the metal implants. The MARS sequence used in this study was provided by the Radiology Department at the Royal National Orthopedic Hospital, Stanmore, United Kingdom. Four sequences were performed per sheep;(1) coronal T1FSE and (2) STIR, (3) axial T2FSE and (4) T1FSE. All sequences taken were of a $4 \mathrm{~mm}$ slice thickness, with a $1 \mathrm{~mm}$ gap. The following pathological changes adjacent to the operated hip were quantified: (1) Joint effusion (Y/N); (2) hip capsule thickness (mm); (3) the presence of a periprosthetic mass $(\mathrm{Y} / \mathrm{N})$; (4) tendon avulsion $(\mathrm{Y} / \mathrm{N}) ;(5)$ muscle atrophy $(\mathrm{Y} / \mathrm{N})$ and (6) fracture $(\mathrm{Y} / \mathrm{N})$. If a periprosthetic mass was present, the following 9 parameters were quantified: (i) maximum diameter of mass (mm); (ii) Fluid filled (F), soft tissue (ST) or mixed (M); (iii) rim: irregular (I) or regular (R); (iv) T2 signal of rim (H / I / L); (v) surrounding ST edema (Y/N); (vi) adjacent bone marrow edema $(\mathrm{Y} / \mathrm{N})$; (vii) cortical/medullary extension of mass $(\mathrm{Y} / \mathrm{N})$; (viii) collection tracking through deep fascia $(\mathrm{Y} / \mathrm{N})$; (ix) pseudotumour grade $(1 / 2 \mathrm{a} / 2 \mathrm{~b} / 3)$. MRI analysis was performed independently by two blinded consultant musculoskeletal radiologists based at the Royal National Orthopedic Hospital, Stanmore, United Kingdom.

\section{Metal debris analysis and ALVAL}

In order to investigate metal debris migration, the following tissues were retrieved from each sheep: (1) left and right synovial tissue adjacent to the hip (in the medial, lateral, anterior, and posterior planes); (2) testes, (3) spleen, (4) kidney, (5) bladder wall, (6) liver, (7) lung, (8) heart, and (9) brain (frontal lobe, parietal lobe, temporal lobe, occipital lobe, and cerebellum). Once collected specimens were immediately fixed in formaldehyde, processed for wax embedding and sections stained with Hematoxylin \& Eosin in the Diagnostic Laboratories at the Royal Veterinary College, Hawkshead, United Kingdom. Synovium was collected from both the left and right (operated) hip immediately adjacent to the implant and in the medial, lateral, anterior and posterior aspects of the joint. In each specimen the synovial lining, inflammatory infiltrate and tissue organization were assessed and an ALVAL score used to determine the level of tissue damage caused following metal debris exposure in each of our experimental groups (4) where scores of 0 (normal tissue) - 10 (ALVAL) were applied. Scores were carried out by two blinded independent assessors.

\section{Statistical analysis}

A Kruskal-Wallis test and post-hoc Mann-Whitney U-test was used to compare data at different time-points and within one experimental group (version 10.1; SPSS Chicago, IL). The non-parametric Mann-Whitney $U$-test was used for statistical analysis between experimental groups. In all cases, the mean \pm standard deviation is presented and $p<0.05$ were considered significant.

\section{RESULTS}

Implants were inserted successfully and all animals remained in good health with no obvious problems with their operated leg throughout the in vivo period. Radiographs taken at 1, 3, $6,9,12$, and 13 months showed articulated and well-fixed joints (Figure 3). Following euthanasia, implants and associated bone and tissue were carefully retrieved. The femoral head of the implant was separated from the stem and the femoral component removed from within the femur. Implants were washed in $100 \%$ industrial methylated spirit, rinsed in tap water followed by distilled water and then dried and packed before further analysis.

\section{Scanning electron microscopy}

Scanning electron microscopy showed the coating to be durable with no signs of delamination or coating loss. Results showed heavily scratched femoral head surfaces in 

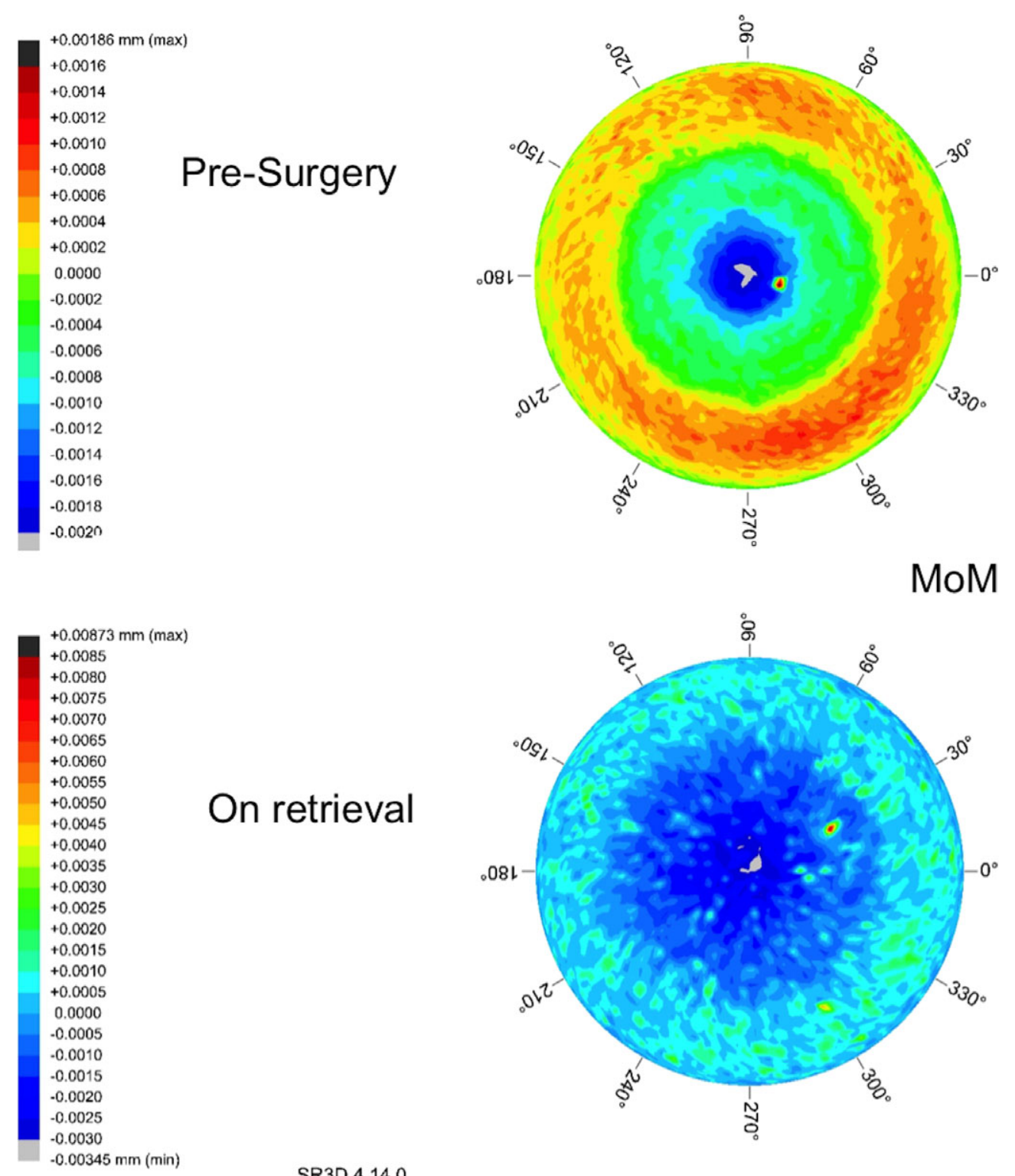

SR3D 4.14.0

FIGURE 6. 3D images showing the surfaces profiles generated from the femoral bearing surface in (A) the SLC-MoM group and (B) the non-coated femoral surface.

the MoP and MoM groups [Figure 4(A,B)]. However, femoral heads coated with SLC appeared less scratched at this time point and similar to the non-implanted pre-operative surface [Figure $4(C, D)]$. Pin-hole defects $(1-3 \mu \mathrm{m}$ in size $)$ were seen within the SLC coating both pre-operatively and following retrieval [Figure $4(C, D)]$. The growth of the SLC coating is influenced by the crystal structure of the underlying substrate, this morphology was seen in the form of discrete crystals $<20 \mu \mathrm{m}$ in size.

\section{Material loss (tapers)}

No volumetric loss of material was measured on either the female or male taper surfaces when all implants were analyzed using the CMM machine and compared between preop and post retrieval values.
Co-ordinate measuring machine (CMM) and Taylor Hobson Talyrond High-Precision Roundness Measuring Machine.

\section{Material loss (bearing surface)}

Acetabular cups. Results obtained pre-operation and at 13 months post-surgery were used to calculate the mean wear loss. Minimal material loss was identified in the two groups investigated and although less material loss was seen in the SLC-MoM group $\left(0.757 \pm 0.453 \mathrm{~mm}^{3}\right)$, no significant difference was found $\left(\mathrm{MoM}=1.015 \pm 0.658 \mathrm{~mm}^{3}\right)$.

Femoral heads. Minimal material loss was identified in the three groups with highest loss measured in the MoM group $\left(-0.631 \pm 0.995 \mathrm{~mm}^{3}\right)$ and lowest in the MoP component $\operatorname{group}\left(-0.135 \pm 0.106 \mathrm{~mm}^{3}\right)\left(\right.$ SLC-MoM $\left.=-0.326 \pm 0.424 \mathrm{~mm}^{3}\right)$. No significant differences were found. Figure 5 shows that in 
TABLE I. Mean Chromium Ion Levels Measured in each Experimental Group Over the 13-Month in Vivo Period (ppb \pm Standard Error)

\begin{tabular}{lcc}
\hline Experimental group & Time (months) post-op & $\mathrm{Cr} p \mathrm{pp}( \pm \mathrm{SE})$ \\
\hline Control & Pre-operative & $0.240 \pm 0.03$ \\
MoM & 1 & $0.490 \pm 0.04$ \\
& 3 & $0.250 \pm 0.04$ \\
& 6 & $0.570 \pm 0.28$ \\
& 12 & $0.791 \pm 0.24$ \\
SLC-MoM & 13 & $0.805 \pm 0.30$ \\
& 1 & $0.300 \pm 0.05$ \\
& 3 & $0.080 \pm 0.05$ \\
& 6 & $0.090 \pm 0.03$ \\
MoP & 12 & $0.226 \pm 0.04$ \\
& 13 & $0.210 \pm 0.03$ \\
& 1 & $0.380 \pm 0.11$ \\
& 3 & $0.100 \pm 0.05$ \\
& 6 & $0.100 \pm 0.00$ \\
& 12 & $0.239 \pm 0.03$ \\
& 13 & $0.213 \pm 0.02$ \\
\hline
\end{tabular}

all instances, the femoral head contour was more uniform postretrieval when compared with that measured pre-implantation.

\section{3-dimensional surface measurements}

Femoral head (taper). The $R_{a}$ measured on the female femoral head taper surface following retrieval showed that the mean values measured in the MoM group were rougher $(0.7067 \pm 0.115 \mu \mathrm{m})$ when compared with both the SLC-MoM $(0.656 \pm 0.21 \mu \mathrm{m})$ and MoP $(0.520 \pm 0.156 \mu \mathrm{m})$ groups, but were less rough when compared with values obtained in the MoM group pre-implantation $(0.7117 \pm 0.124 \mu \mathrm{m})$. Postretrieval, the roughness of tapers in the MoP and SLC-MoM groups were also less when compared with pre-implantation values $(0.593 \pm 0.136 \mu \mathrm{m}$ and $0.637 \pm 0.208 \mu \mathrm{m}$, respectively). No significant differences between groups were found. Similar results were found when $R_{z}$ values were compared between groups.

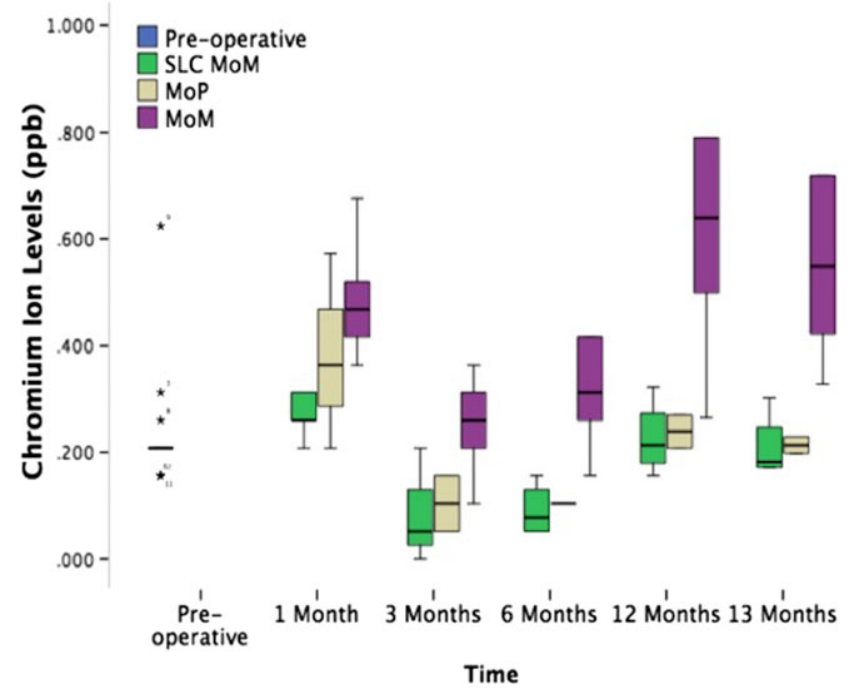

FIGURE 7. Chromium levels obtained in all groups pre-operatively and at $1,3,6,12$, and 13 months post-operatively.
Femoral stem (neck) taper. Results showed that the mean surface roughness values $\left(R_{a}\right)$ measured in the MoM group $(0.707 \pm 0.115 \mu \mathrm{m})$ at 13 months post-surgery increased when compared with both the SLC-MoM $(0.656 \pm 0.219 \mu \mathrm{m})$ and MoP $(0.520 \pm 0.156 \mu \mathrm{m})$ groups, but were less rough when compared with values obtained in the MoM group preimplantation $(0.712 \pm 0.124 \mu \mathrm{m})$. After retrieval the roughness of tapers in the MoP and SLC-MoM groups were less when compared with pre-implantation values $(0.593 \pm 0.136 \mu \mathrm{m}$ and $0.637 \pm 0.210 \mu \mathrm{m}$, respectively). No significant differences between groups were found and similar results were seen when $R_{z}$ values were compared.

Femoral head (bearing surface). A mean $\mathrm{R}_{\mathrm{a}}$ of $0.131 \pm 0.055 \mu \mathrm{m}$ was measured on the SLC femoral head surface pre-implantation and this surface was significantly rougher when compared to the non-coated MoM and MoP bearing surfaces at this time point $(0.015 \pm 0.001 \mu \mathrm{m})$ (Figure 6). At 13 months post-implantation, surface roughness did not change and no significant difference in $R_{a}$ was measured when compared with pre-implantation values. At 13 months post-operatively, significantly increased $R_{a}$ values were measured in the SLC-MoM group $(0.157 \pm 0.022 \mu \mathrm{m})$ when compared with both MoM $0.091 \pm 0.165 \mu \mathrm{m}$; $p=0.055)$ and MoP $(0.016 \pm 0.004 \mu \mathrm{m} ; p=0.046)$ groups. No other significant differences were found. When peak-tovalley height $\left(R_{z}\right)$ was measured on the bearing surface of femoral heads retrieved 13 months post-surgery, results showed that significantly increased values were measured in the SLC-MoM group $(5.595 \pm 0.469 \mu \mathrm{m})$ when compared with both MoM (1.28 $\pm 0.49 \mu \mathrm{m} ; p=0.006)$ and $\mathrm{MoP}$ $(0.79 \pm 0.039 \mu \mathrm{m} ; p=0.046)$ components. Results also showed an increased peak-to-valley height in the MoM group when compared with the MoP group ( $p=0.053)$.

Acetabular cup (bearing surface). At time zero, SLC implants had a significantly rougher surface when compared

TABLE II. Mean Cobalt lon Levels Measured in Each Experimental Group Over the 13-Month in Vivo Period $(p p b \pm$ Standard Error)

\begin{tabular}{lcc}
\hline Experimental group & Time (months) post-op & Co ppb $( \pm \mathrm{SE})$ \\
\hline Control & Pre-operative & $1.190 \pm 0.20$ \\
MoM & 1 & $2.400 \pm 0.53$ \\
& 3 & $1.470 \pm 0.19$ \\
& 6 & $4.430 \pm 2.25$ \\
& 12 & $4.390 \pm 2.91$ \\
SLC-MoM & 13 & $2.720 \pm 1.54$ \\
& 1 & $1.600 \pm 0.53$ \\
& 3 & $1.190 \pm 0.17$ \\
& 6 & $1.060 \pm 0.10$ \\
MoP & 12 & $0.690 \pm 0.06$ \\
& 13 & $0.670 \pm 0.08$ \\
& 1 & $1.850 \pm 0.89$ \\
& 3 & $0.170 \pm 0.97$ \\
& 6 & $1.740 \pm 1.15$ \\
& 12 & $0.780 \pm 0.34$ \\
& 13 & $0.950 \pm 0.45$ \\
\hline
\end{tabular}




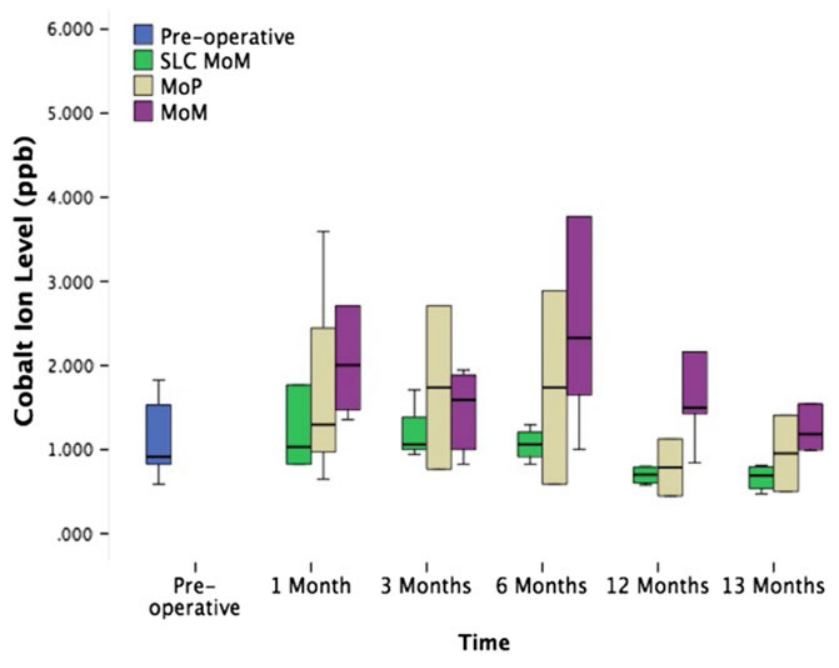

FIGURE 8. Cobalt levels obtained in all groups at pre-operatively and at 1 and 3 months post-operatively.

with the non-coated bearings. There was no significant difference in $R_{a}$ or $R_{z}$ between the pre-implantation and retrieved SLC-MoM implants. At 13 months post-surgery, results showed significantly increased $R_{a}$ values in the SLCMoM group $(0.022 \pm 0.006 \mu \mathrm{m})$ when compared with MoM components $(0.049 \pm 0.016 \mu \mathrm{m} ; p=0.045)$. Results also showed significantly increased peak-to-valley height $\left(R_{Z}\right)$ values in the SLC-MoM group $(5.806 \pm 1.912 \mu \mathrm{m})$ when compared with MoM components (1.305 $\pm 0.603 \mu \mathrm{m}$; $p=0.006)$.

\section{Chromium and cobalt metal ion analysis}

Chromium ion levels. The mean $\mathrm{Cr}$ ion level measured within blood prior to surgery was $0.24 \pm 0.03 \mathrm{ppb}$ (Table I and Figure 7). Results showed significantly lower levels of $\mathrm{Cr}$ in the SLC-MoM group when compared with the MoM group at $1(p=0.029), 3(p=0.024), 6(p=0.013)$, $12(p=0.019)$, and $13(p=0.010)$ months post operatively. A significantly decreased amount of $\mathrm{Cr}$ ions were measured in the MoP group when compared with the MoM group at $6(p=0.043)$ and 13 months $(p=0.046)$ post operatively. There was no significant difference between MoP and SLCMoM at all time-points. When compared within groups, results over time showed a significant decrease in $\mathrm{Cr}$ ion levels in the MoM and SLC-MoM groups between the 1- and 3 -month post-operative period $(p=0.005$ and $p=0.013$, respectively). In the SLC-MoM group only, significantly decreased levels of $\mathrm{Cr}$ were also measured at 6 months when compared with blood collected 1 monthly post operatively ( $p=0.009$ ). In the MoM group, significantly higher $\mathrm{Cr}$ ion levels were measured in blood at 12 and 13 months post operatively when compared with levels found at 3 months ( $p=0.010$ and $p=0.006$, respectively). No other significant differences were found.

Cobalt ion levels. The mean Co ion level measured within blood prior to surgery was $1.19 \pm 0.20 \mathrm{ppb}$ (Table II). This measurement increased in all groups at 1-month post- surgery, before reducing in all groups at 3 months postsurgery (Figure 8). In all groups, Co ion levels decreased at 12 and 13 months post-surgery, however in the MoM group, levels remained elevated when compared with the SLC-MoM and MoP bearing groups. Results showed that significantly higher Co ion levels were measured in animals with a MoM bearing at 1 and 6 months when compared with levels seen pre-operatively ( $p=0.010$ and $p=0.006$, respectively). At 12 and 13 months, significantly lower levels of cobalt were measured in the SLC-MoM group when compared with preoperative values ( $p=0.045$ at both time-points), 1 month ( $p=0.020$ at both time-points), and 3 months ( $p=0.021$ at both time-points). No other significant differences were found.

Comparing cobalt blood levels between groups showed significantly higher levels of Co ions in the MoM group when compared with the SLC-MoM group at $6(p=0.042)$, $12(p=0.011)$, and $13(p=0.011)$ months post-operatively. Results showed no other significant differences when the groups were compared at these three time-points. No significant difference was found when the MoP and SLC-MoM groups were compared.

MRI results. Results showed that none of the animals had joint effusion, a periprosthetic mass, surrounding soft tissue or adjacent bone marrow edema. None of the animals had tendon avulsions and none a fractured femur. All except two animals (both in the SLC-MoM group) showed adjacent muscle atrophy within the gluteal muscles, possibly due to postsurgical trauma. The synovium could not be imaged in any animal due to metal artifact and therefore capsule thickness was not measured.

\section{Metal debris analysis}

Post-mortem observations. In all of the animals investigated, dissection of the right hip showed no signs of abnormal tissue. No pseudotumours, necrotic masses or pockets containing exudate were observed. However, in all animals a thick fibrous capsule was found surrounding the articulation. Fragments of fibrin were found within the capsular tissue

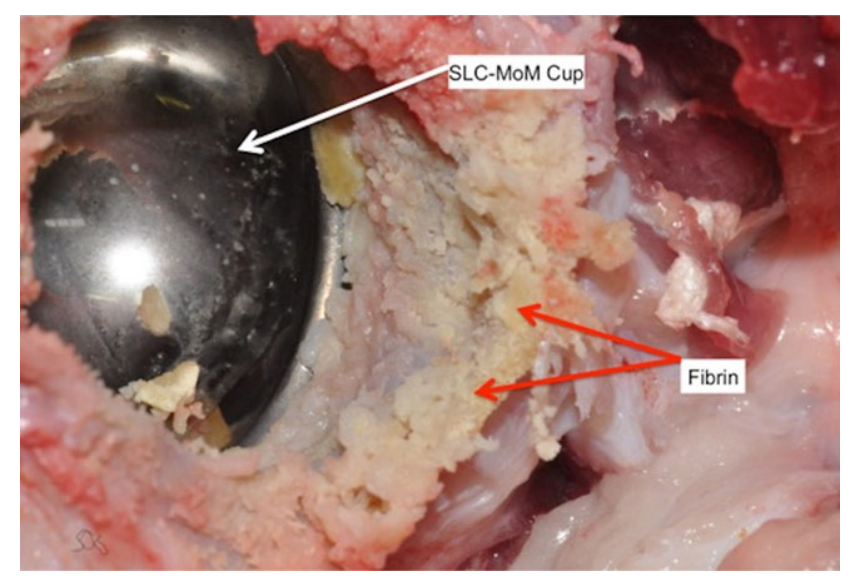

FIGURE 9. Fibrin within tissue adjacent to a MoM bearing at 13 months post-surgery. 


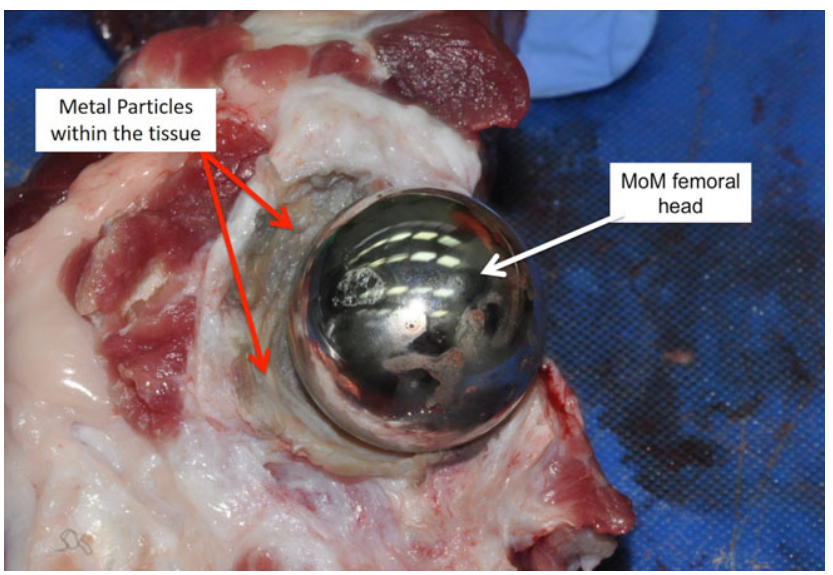

FIGURE 10. Metal particles within tissues adjacent to the femoral head in the MoM bearing group at 13 months post-surgery.

and immediately adjacent to the joint in a total of three animals in both the SLC MoM group and MoM groups (Figure 9). Additionally and in one animal discolored tissue, which suggested the presence of metal particles, occurred in the MoM group and was seen adjacent to the femoral head (Figure 10).

Histological analysis of retrieved tissues using the ALVAL score. Following examination using light microscopy, no evidence of metal debris was observed in samples taken from the testes, spleen, lung, kidney, heart, bladder wall, brain, and liver in all animals investigated in this study. The synovial tissue retrieved from the left unoperated hip joint in all animals also did not contain any metallic debris or damage and each of these tissues scored " 0 " on the ALVAL scoring system.

Wear debris was observed both within the tissues and following phagocytosis within macrophages and foreign body giant cells in both the SLC-MoM and MoM groups (Figure 11). No metallic wear debris was observed within samples obtained from MoP specimens. Results showed that a significantly higher ALVAL score was measured in the MoM group (4.67 \pm 0.14 ) (Figure 12) when compared with the values measured in tissue adjacent to SLC-MoM implants $(2.56 \pm 0.21 ; p=0.010)$. Tissue inflammation adjacent to the implant was not observed in any of the experimental groups investigated and the increased ALVAL score measured in the MoM group was as a result of an increased loss of synovium with associated fibrin attachment, increased infiltration of macrophages often within perivascular aggregates and a marked loss in the normal arrangement of the tissue with relatively thick acellular areas observed throughout.

\section{DISCUSSION}

In vitro preclinical testing failed to predict the biological effect of wear generated at the bearing surfaces and for this reason, the additional use of preclinical in vivo models may provide important information when establishing the performance of new orthopedic devices. However, orthopedics presents many challenges for preclinical testing since the forces, vectors and geometry of the joints in humans are not replicated in any other species. An ovine arthroplasty model offers several advantages over smaller animal models in that they are large enough to be able to use representative functional implants with a large volume and surface area, whereas smaller animals make this very difficult. Although the forces and the motions of an ovine hip replacement are different compared to humans they are able to restore normal hip joint motion and are subject to wear of articulating surfaces. ${ }^{19}$ In this study, an ovine model was developed to screen and discern the performance of different bearing materials over a 13-month post-operative period. Our hypothesis was that a SLC applied to the femoral head and acetabular cup surfaces would result in a reduction in volumetric wear, a decrease in blood chromium and cobalt ion levels and reduce any potential ARMDs. Our results demonstrated the potential of a MoM articulation to release metal particles and significantly increase the circulating level of $\mathrm{Cr}$ and Co ions and ALVAL score. We conclude that this model may have the potential to not only predict wear of articular surfaces but also to assess the biological response to any

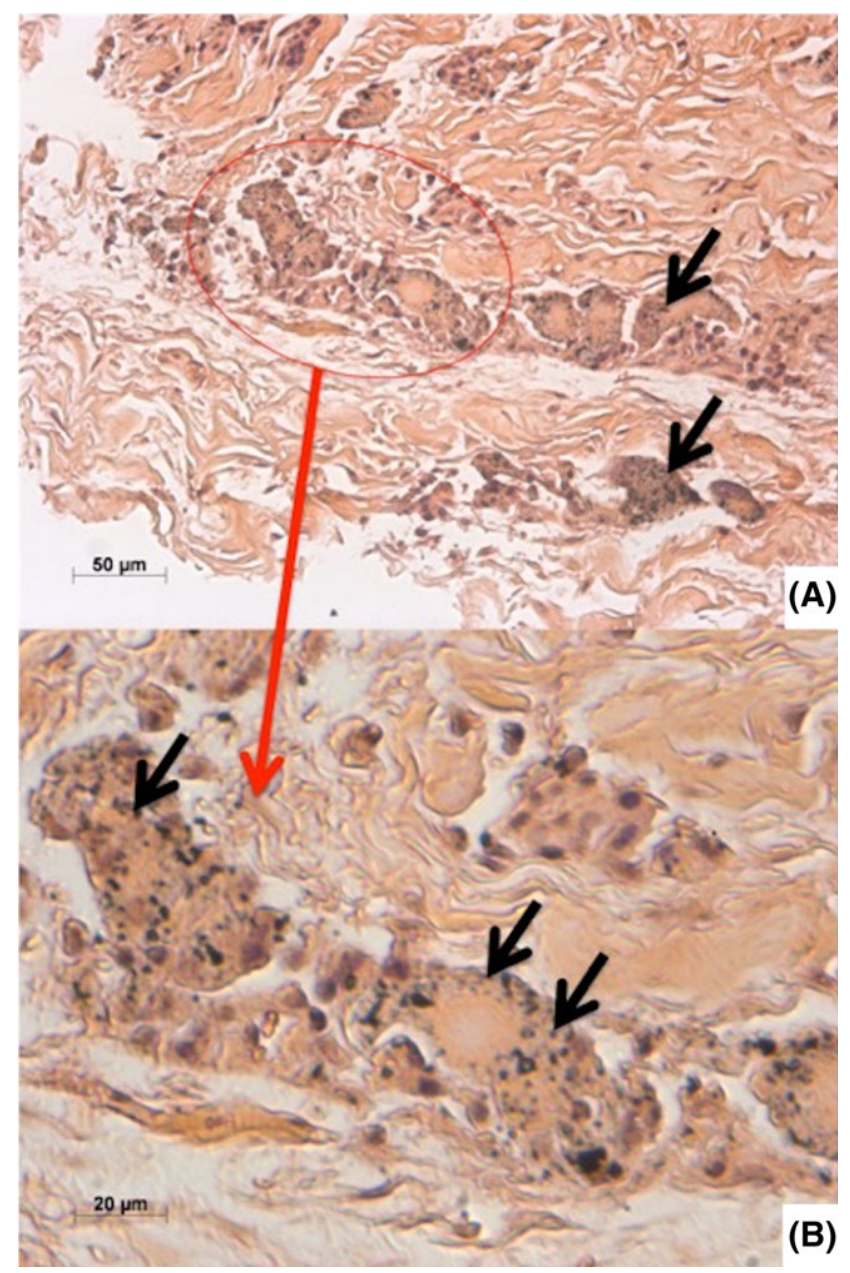

FIGURE 11. A light photomicrograph showing metallic debris within the cell cytoplasm. Magnification $(A) \times 20 ;(B) \times 50$. 


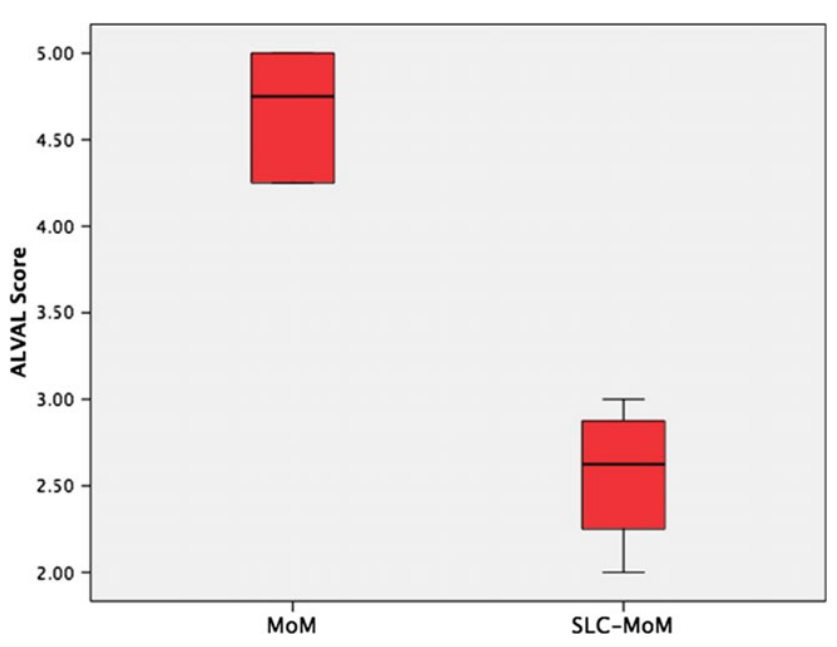

FIGURE 12. The ALVAL scores obtained from the synovial tissues adjacent to the implant in each of the groups at 13 months post-surgery.

wear debris that is released and may be used in the future to predict the performance of new implant materials used for articulation. Although the implants were inserted in an optimal position, it would be possible to deliberately misplace the cups to reproduce edge wear, which is considered to be important with hard on hard bearings. This would increase the wear rate and consequently the biological burden of the wear debris.

This study showed that the surface roughness of SLC surfaces pre-implantation was significantly increased when compared with a non-coated polished metal surface (in both femoral heads and acetabular cups). Theoretical and experimental studies have shown that a reduction in surface roughness of MoM and MoP bearings can lead to reduced wear, due to improvements in the lubrication and abrasion conditions. $^{20,21}$ However, no difference in measured wear were seen when the coated and non-coated groups were compared in this study. Scanning electron microscopy showed the coating to be durable over the 13-month period, with no signs of delamination, cracking or coating loss. Pin hole defects were seen throughout the SLC coating in both pre-operative samples and those following retrieval. Formation of these defects were deemed to be associated with artifact generated during the coating process and defect density was not quantified in this study. Results also showed that post-retrieval, the $R_{a}$ and $R_{z}$ values increased in the MoM group when compared with retrieved MoP femoral heads. SEM demonstrated areas of multiple scratches on the heads and cups of retrieved components in the MoM and MoP groups, however scratches were not evident on the SLC surface. Minimal material loss through wear was seen both within the male and female tapers on the femoral stem and femoral neck respectively and also from the head and cup bearing surfaces in all groups. No material loss was measured from tapers in any of the experimental groups investigated, however it should be noted only four measurements were made per taper and full measurements that cover the entire taper surface would give a more accurate representation of wear in this region. No significant differences in $R_{a}$ and $R_{z}$ values were seen when taper surface roughness was compared between groups although in all groups, the roughness on both the male and female taper had decreased when pre-implantation data was compared with post-retrieval results. Polishing of the taper surface may be due to relative movement however, usually fretting corrosion at the trunion results in an imprinting effect on the female cobalt chrome surface but this was not seen in the explants from the ovine model and it is therefore assumed that this reduction in roughness was due to flattening of the surface peaks during taper assembly and impaction. This combined with the indication of no material loss measured at each taper, suggests that in this study any adverse reactions to debris may have been mainly associated with particles released from the articulation and not from the taper.

Superlattice ceramic coating showed significantly lower levels of both chromium and cobalt metal ions levels within blood over the 13-month period. The authors can find no other studies that report on the wear and metal ion release following wear testing of a MoM bearing surface with a $\mathrm{CrN} / \mathrm{NbN}$ coating however, previous in vitro simulator studies have investigated a CrN coating on a MoM bearing surface and reported a reduction in wear and ion levels. ${ }^{21,22}$ These studies concluded that the reduction in wear in the coated group, was due to an increased hardness resulting in an increased resistance to abrasive wear where the $\mathrm{CrN}$ coated bearings were behaving like a ceramic-on-ceramic bearing. $\mathrm{CrN} / \mathrm{NbN}$ is chemically inert and biocompatible and therefore a further advantage in the use of this coating is the improved biocompatibility, reduced corrosion and reduced cytotoxicity of the wear particles produced. The lower ion levels may therefore be attributed to the decreased wear rates and reduced corrosion of the implant surfaces ${ }^{23}$ combined possibly with the increased resistance to degradation of the released wear products and this is a major advantage of the SLC investigated. The size and shape of the wear particles produced within each of the bearing combinations were not investigated. However, a previous study ${ }^{22}$ showed that wear particles generated by the $\mathrm{CrN}$ coated bearing surfaces were similar in size and shape to those produced by a MoM bearings. A previous study found $\mathrm{CrN}$ to be cytotoxic to fibroblast and macrophage cell lines at concentrations of $50 \mu \mathrm{m}^{3} /$ cell and above, compared with $0.5 \mu \mathrm{m}^{3} /$ cell for $\mathrm{CoCr}^{24}$ This may lead to a reduction in the risk of tissue necrosis and ARMD as found in this study.

Histological assessment showed no metallic debris biodistribution within tissues and organs in any experimental group, except for debris observed within synovial tissue immediately adjacent to the implant bearing. The ALVAL scores measured were relatively low however discoloration was observed within tissue adjacent to an implant in the MoM group and a significantly lower ALVAL score measured in the SLC-MoM group when compared with MoM components. ALVAL results also showed that SLC-MOM components obtained a similar score to tissues in the MoP sample group. Cyst and pseudotumor formation is often associated with metallic debris adjacent to MoM bearings, however MRI analysis showed no tissue abnormalities in any of the 
animals investigated in this study. The blood metal ion levels obtained were relatively low when compared to that reported in the literature. A systematic review by Campbell et al. $^{25}$ showed that when serum chromium and/or cobalt levels exceeded $7 \mathrm{ppb}$, ARMD was observed in patients. Other authors have identified lower metal levels associated with the ARMD risk and Bisschop et al. ${ }^{26}$ found that the chance of having a pseudotumor was significantly higher in patients with an elevated serum cobalt level of $>5.5 \mathrm{ppb}$. Bisschop's study also showed that an increase in cobalt or chromium ions of $1 \mathrm{ppb}$ increased the chance of pseudotumor formation by an odds ratio of 1 . It was unclear in this study as why the mean pre-operative value of Co ions was above $1 \mathrm{ppb}$. Measures were taken when collecting, storing and analyzing blood samples to limit contamination and so this may be an anomaly associated with the ovine model. The highest mean level of $\mathrm{Cr}$ and Co ions measured in this study was $0.805 \pm 0.30 \mathrm{ppb}$ and $4.43 \pm 2.25 \mathrm{ppb}$, respectively and both were measured in the non-coated MoM group. Although necrosis was observed adjacent to the hip articulation in this group, no pseudotumor formation or cysts were evident. There is no clear evidence reported of a dose-response to metal debris, however higher wear is associated with a higher frequency of adverse reactions ${ }^{25}$ and therefore a limitation of our animal model may be time. A longer in vivo period may be necessary to generate sufficient metal debris and ion release to cause fluid collections and/or pseudotumor formation and it is not known if ovine physiology differs from human physiology in its reaction to debris. However, previously an ovine model where the femoral head was deliberately roughed in order to create polyethylene wear, was shown to cause osteolysis. ${ }^{19,27}$

\section{CONCLUSIONS}

This study used a repeatable and reproducible total hip replacement ram model to investigate the effects of the wear and toxicity of released debris from different implant bearing materials in vivo. A ceramic $\mathrm{CrN} / \mathrm{NbN}$ coating was durable with no signs of delamination, coating loss or adverse reactions. Although a decrease in volumetric wear was not measurable in this study, our hypothesis can be partly accepted as a significant reduction in $\mathrm{Cr}$ and Co ion release from the underlying substrate and a decrease in the ALVAL score were achieved following coating application onto the articulating surfaces in metal-on-metal hip replacement surgery. This coating offers the opportunity to increase the survival of joint replacements by reducing metal ion release and may be suitable for patients that have metal ion sensitivities.

\section{ACKNOWLEDGMENTS}

This work was supported by ZimmerBiomet UK. The authors would like to thank Gillian Hughes at the Royal Veterinary College for animal husbandry. Conflicts of Interest: The authors HW, TM and IK are employed by ZimmerBiomet, UK. All other authors have nothing to declare.

\section{REFERENCES}

1. Smith AJ, Dieppe P, Howard PW, Blom AW. National joint registry for England and Wales. Failure rates of metal-on-metal hip resurfacings: Analysis of data from the National Joint Registry for England and Wales. Lancet 2012;380:1759-1766.

2. Smith AJ, Dieppe P, Vernon K, Porter M, Blom AW. National joint registry of England and Wales. Failure rates of stemmed metal-onmetal hip replacements: Analysis of data from the national joint registry of England and Wales. Lancet 2012;379:1199-1204.

3. De Smet K, De Haan R, Calistri A, Campbell PA, Ebramzadeh E, Pattyn C, Gill HS. Metal ion measurement as a diagnostic tool to identify problems with metal-on-metal hip resurfacing. J Bone Joint Surg 2008;90(Suppl4):202-208.

4. Hart AJ, Matthies A, Henckel J, llo K, Skinner J, Noble P. Understanding why metal-on-metal hip arthroplasties fail. A comparison between patients with well-functioning and revised Birmingham hip resurfacing arthroplasties. J Bone Joint Surg Am 2012;94:1-10.

5. Briggs TWR, Hanna SA, Kayani B, Tai S, Pollock RC, Cannon SR, Blunn GW, Carington RWJ. Metal-on-polyethylene versus metal-onmetal bearing surfaces in total hip arthroplasty: A prospective randomised study investigating metal ion levels and chromosomal aberrations in peripheral lymphocytes. Bone Joint J 2015;97B: 1183-1191.

6. Doorn PF, Campbell PA, Worrall J, Benya PD, McKellop HA, Amstutz HC. Metal wear characterization from metal on metal total hip replacements: Transmission electron microscopy study of periprosthetic tissues and isolated particles. J Biomed Mater Res 1988; 42:103-111.

7. Gilbert JL, Buckley CA, Jacobs JJ, Bertin KC, Zernich MR. Intergranular corrosion-fatigue failure of cobalt-alloy femoral stems. J Bone Joint Surg Am 1994;76:110-115.

8. Goldberg JR, Buckley CA, Jacobs JJ, Gilbert JL. Modularity of Orthopaedic Implants ASTM STP 1301 Corrosion Testing of Modular Hip Implants; 1997. pp. 157-176.

9. Collier JP, Mayor MB, Jensen RE. Mechanisms of failure of modular prostheses. Clin Orthop Relat Res 1992;285:129-139.

10. Fricker DC, Shivanathan R. Fretting corrosion studies of universal femoral head prostheses and cone taper spigots. Biomaterials 1990;11:495-500.

11. Gilbert JL, Buckley CA, Jacobs JJ. In vivo corrosion of modular hip prosthesis components in mixed and similar metal combinations. The effect of crevice, stress, motion and alloy coupling. J Biomed Mater Res 1993;27:1533-1554.

12. van der Veen $\mathrm{HC}$, Reininga IHF, Zijlstra WP, Boomsma MF, Bulstra SK, van Raay JJAM. Pseudotumour incidence, cobalt levels and clinical outcome after large head metal-on-metal and conventional metal-on-polyethylene total hip arthroplasty. Bone Joint $\mathrm{J}$ 2015;97B:1481-1487.

13. Campbell $P$, Ebramzadeh E, Nelson S, Takamura K, De Smet $K$, Amstutz HC. Histological features of pseudotumour-like tissue from metal-on-metal hips. Clin Orthop Relat Res 2010;468(9): 2321-2327.

14. Natu S, Sidaginamale RP, Gandhi J, Langton DJ, Nargol AV. Adverse reactions to metal debris: Histopathological features of periprosthetic soft tissue reactions seen in association with failed metal-on-metal hip arthroplasties. J Clin Pathol 2012;65:409-418.

15. Girard J, Lavigne M, Vendittoli PA, Roy AG. Biomechanical reconstruction of the hip: A randomised study comparing total hip resurfacing and total hip arthroplasty. J Bone Joint Surg Br 2006;88:721-726.

16. McMinn D, Daniel J. History and modern concepts in surface. Proc Inst Mech Eng H 2011;220:239-251.

17. Daniel J, Pynsent PB, McMinn DJ. Metal-on-metal resurfacing of the hip in patients under the age of 55 years with osteoarthritis. J Bone Joint Surg Br 2004;86B:177-184.

18. Hovsepian PE, Ehiasarian AP, Purandare $\mathrm{Y}$, Sugumaran AA, Marriott T, Khan I. Development of superlattice $\mathrm{CrN} / \mathrm{NbN}$ coatings for joint replacements deposited by high power impulse magnetron sputtering. J Mater Sci Mater Med 2016;27(9):147.

19. Coathup MJ, Blackburn J, Goodship AE, Cunningham JL, Smith T, Blunn GW. Role of hydroxyapatite coating in resisting wear particle migration and osteolysis around acetabular components. Biomaterials 2005;26(19):4161-4169.

20. Williams $S$, Isaac $G$, Hatto $P$, Stone $M H$, Ingham E, Fisher J. Comparative wear under different conditions of surface engineered 
metal-on-metal bearings for total hip arthroplasty. J Arthroplasty 2004;19(8):112-117.

21. Leslie IJ, Williams S, Brown C, Anderson J, Isaac G, Hatto P, Ingham E, Fisher J. Surface engineering: A low wearing solution for metal-on-metal hip surface replacements. J Biomed Mater Res Part B Appl Biomater 2009;90B:558-565.

22. Fisher J, Hu XQ, Stewart TD, Williams $\mathrm{S}$, Tipper JL, Ingham $\mathrm{E}_{,}$ Stone $\mathrm{MH}$, Davies C, Hatto P, Bolton J, Riley M, Hardaker C, Isaac G, Berry G. Wear of surface engineered metal-on-metal hip prostheses. J Mater Sci Mater Med 2004;15:225-235.

23. Bolton $\mathrm{J}, \mathrm{Hu} X$. In vitro corrosion testing of PVD coatings applied to surgical grade CoCrMo alloy. J Mater Sci Mater Med 2002;13: 567-574.

24. Williams S, Tipper JL, Ingham E, Stone MH, Fisher J. In vitro analysis of the wear, wear debris and biological activity of surface-engineered coatings for use in metal-on-metal total hip replacements. Proc Inst Mech Eng H 2003;217:155-163.

25. Campbell PA, Kung MS, Hsu AR, Jacobs JJ. Do retrieval analysis and blood metal measurements contribute to our understanding of adverse local tissue reactions? Clin Orthop Relat Res 2014;472: 3718-3727.

26. Bisschop R, Boomsma MF, Van Raay JJ, Tiebosch AT, Maas M, Gerritsma CL. High prevalence of pseudotumours in patients with a Birmingham hip resurfacing prosthesis: A prospective cohort study of one hundred and twenty-nine patients. J Bone Joint Surg Am 2013;95:1554-1560.

27. Bhumbra RP, Walker PS, Berman AB, Emmanual J, Barrett DS, Blunn GW. Prevention of loosening in total hip replacements using guided bone regeneration. Clin Orthop Relat Res 2000;372: 192-204. 